\title{
Overcoming Trastuzumab Resistance in HER2-Overexpressing Breast Cancer Cells by Using a Novel Celecoxib-Derived Phosphoinositide-Dependent Kinase-1 Inhibitor
}

\author{
Ping-Hui Tseng, Yu-Chieh Wang, Shu-Chuan Weng, Jing-Ru Weng, Chang-Shi Chen, \\ Robert W. Brueggemeier, Charles L. Shapiro, Ching-Yu Chen, Sandra E. Dunn, \\ Michael Pollak, and Ching-Shih Chen
}

Division of Medicinal Chemistry and Pharmacognosy, College of Pharmacy (P.-H.T., Y.-C.W., S.-C.W., C.-S.C., R.W.B., C.-S.C.), and Division of Hematology and Oncology, Department of Internal Medicine (C.L.S.), the Ohio State University, Columbus, Ohio; Department of Biological Science and Technology, China Medical University and Hospital, Taichung, Taiwan (J.-R.W.); Department of Family Medicine, College of Medicine, National Taiwan University, and the Gerontology Research Division, the National Health Research Institutes, Taipei, Taiwan (C.-Y.C.); British Columbia Research Institute for Children's and Women's Health, Department of Pediatrics, University of British Columbia. Vancouver, British Columbia, Canada (S.E.D.); and Department of Oncology, Jewish General Hospital, and McGill University, Montreal, Canada (M.P.)

Received February 28, 2006; accepted August 3, 2006

\begin{abstract}
Although trastuzumab has been successfully used in patients with HER2-overexpressing metastatic breast cancer, resistance is a common problem that ultimately culminates in treatment failure. In light of the importance of Akt signaling in trastuzumab's antitumor action, we hypothesized that concurrent inhibition of Akt could enhance trastuzumab sensitivity and moreover reverse the resistant phenotype in HER2-positive breast cancer cells. Based on our finding that celecoxib mediates antitumor effects through the inhibition of phosphoinositide-dependent kinase-1 (PDK-1)/Akt signaling independently of cyclooxygenase-2 (COX-2), we used celecoxib as a scaffold to develop a COX-2-inactive PDK-1 inhibitor, 2-amino- $N$-[4-[5-(2-phenanthrenyl)-3-(trifluoromethyl)- $1 \mathrm{H}$ pyrazol-1-yl]phenyl]-acetamide (OSU-03012). Here, we investigated the effect of OSU-03012 on trastuzumab-mediated apoptosis in four breast cancer cell lines with different HER2 expression and trastuzumab-resistance status, including MDA-
\end{abstract}

MB-231, BT474, SKBR3, and insulin-like growth factor-I receptoroverexpressing SKBR3 (SKBR3/IGF-IR). Effects of trastuzumab and OSU-03012, individually or in combination, on cell viability and changes in pertinent biomarkers including HER2 expression, phosphorylation of Akt, p27 $7^{k i p 1}$, and the PDK-1 substrate p70 ${ }^{\text {S6K }}$ were assessed. OSU-03012 alone was able to trigger apoptosis in all cell lines with equal potency $\left(\mathrm{IC}_{50}=3-4 \mu \mathrm{M}\right)$, suggesting no cross-resistance with trastuzumab. Medium dose-effect analysis indicates that OSU-03012 potentiated trastuzumab's antiproliferative effect in HER2-positive cells, especially in SKBR3/IGF-IR cells, through the down-regulation of PDK-1/Akt signaling. This synergy, however, was not observed in HER2negative MDA-MB-231 cells. This combination treatment represents a novel strategy to increase the efficacy of trastuzumab and to overcome trastuzumab resistance in the treatment of HER2-positive breast cancer.
Trastuzumab (Herceptin), a humanized monoclonal antibody targeting the extracellular domain of the tyrosine kinase receptor HER2, has been used in combination chemotherapy

This work was supported by Public Health Service grant CA94829 and Susan G. Komen Breast Cancer Foundation grant BCTR0504187 (to C.S.C.), and translation acceleration grants from the Canadian Breast Cancer Research Initiative (to S.E.D. and M.P.)

Article, publication date, and citation information can be found at http://molpharm.aspetjournals.org.

doi:10.1124/mol.106.023911. for the treatment of HER2-overexpressing metastatic breast cancer, an aggressive form of the disease with poor prognosis. More recently, the results of three large adjuvant trials demonstrate that trastuzumab in combination with chemotherapy significantly increased disease-free survival for women with early-stage HER2-positive breast cancer. Despite these advances, the clinical benefit of trastuzumab is compromised by the facts that not all HER2-overexpressing cancers respond clinically to the treatment and that some breast can-

ABBREVIATIONS: PI3K, phosphatidylinositol 3-kinase; PDK-1, phosphoinositide-dependent kinase-1; IGF-IR, insulin-like growth factor-I receptor; SKBR3/IGF-IR, insulin-like growth factor-I receptor-overexpressing SKBR3; CDK, cyclin-dependent kinase; COX-2, cyclooxygenase-2; MTT, 3-(4,5-dimethylthiazol-2-yl)-2,5-diphenyl-2H-tetrazolium bromide; $\mathrm{Cl}$, combination index; PARP, poly(ADP-ribose) polymerase; OSU-03012, 2-amino- $N$-[4-[5-(2-phenanthrenyl)-3-(trifluoromethyl)-1H-pyrazol-1-yl]phenyl]-acetamide; DMEM/F12, Dulbecco's minimal essential medium/ Ham's F-12; FBS, fetal bovine serum; DMSO, dimethyl sulfoxide; TBST, Tris-buffered saline/Tween 20; PBS, phosphate-buffered saline; Hsp90, 90-kDa heat shock protein; ELISA, enzyme-linked immunosorbent assay; PI, propidium iodide; LY294002, 2-(4-morpholinyl)-8-phenyl-1(4H)benzopyran-4-one hydrochloride. 
cers develop resistance to this molecularly targeted drug after initial response. Evidence indicates that trastuzumab mediates antiproliferative effects in HER2-positive cells by facilitating HER2 degradation (Yarden, 2001), down-regulation of phosphatidylinsitol 3-kinase (PI3K)/Akt signaling (Clark et al., 2002), and increases in the expression of the cyclin-dependent kinase inhibitor p2 $7^{\text {kip } 1}$. Therefore, HER2positive breast cancer cells are able to evade the antiproliferative action of trastuzumab by adopting mechanisms to counter these antiproliferative mechanisms (Hynes and Lane, 2005), including up-regulation of insulin-like growth factor-I receptor (IGF-IR) expression (Lu et al., 2001), loss of PTEN function (Nagata et al., 2004), and down-regulation of the cyclin-dependent kinase (CDK) inhibitor p27 ${ }^{\text {kip } 1}$ expression (Nahta et al., 2004). In light of the important role of Akt signaling in the antitumor action of trastuzumab (Yakes et al., 2002), these mechanistic findings provide a rationale for the use of an Akt signaling inhibitor to enhance the sensitivity to trastuzumab and, more important, to overcome trastuzumab resistance (Arteaga, 2003; Nagata et al., 2004).

On the basis of our finding that the cyclooxygenase-2 (COX-2) inhibitor celecoxib mediates apoptosis in cancer cells, in part, by blocking phosphoinositide-dependent kinase-1 (PDK-1)/Akt signaling independently of COX-2 inhibition, we have used celecoxib as a scaffold to develop a novel class of PDK-1 inhibitors devoid of COX-2 activity (Zhu et al., 2004). The novel compound OSU-03012 exhibits high potency in deactivating Akt and inducing apoptosis at low micromolar concentrations in cancer cells through PDK-1 inhibition. Because PDK-1 is a proximal mediator of PI3K signals, PDK-1 inhibitors target a large portion of the PI3K/Akt pathway and can potentially offer an effective treatment (Crowder and Ellis, 2005). This celecoxib-derived PDK-1 inhibitor has been demonstrated to be effective in inhibiting the proliferation of a panel of breast cancer cell lines (Kucab et al., 2005) and is currently undergoing preclinical testing under the Rapid Access to Intervention Development program at the National Cancer Institute.

We hypothesized that concurrent inhibition of PDK-1/Akt signaling would sensitize HER2-positive breast cancer cells, even those with the resistant phenotype, to trastuzumab's antiproliferative effect. A similar strategy has been used to overcome imatinib mesylate resistance in Bcr-Abl mutant cell lines (Tseng et al., 2005). Here, we demonstrate that OSU-03012 interacted synergistically with trastuzumab to enhance cell killing in two HER2-positive breast cancer cells (BT474 and SKBR3) and to reverse the resistant phenotype in SKBR3 cells that overexpress IGF-IR (SKBR3/IGF-IR). This synergistic effect, however, was not observed in MDAMB-231 cells that lack HER2 expression.

\section{Materials and Methods}

Cell Culture and Reagents. We used the following four breast cancer cell lines to assess the impact of the PDK-1 inhibitor OSU03012 on the sensitivity to the antiproliferative action of trastuzumab: low HER2-expressing MDA-MB-231, HER2-positive BT474, SKBR3, and HER2-positive and trastuzumab-resistant SKBR3/ IGF-IR (Lu et al., 2001). Cells were grown in $75-\mathrm{cm}^{2}$ plastic tissue culture flasks at $37^{\circ} \mathrm{C}$ in a humidified incubator in Dulbecco's minimal essential medium/Ham's F-12 (DMEM/F12; 1:1) medium containing $10 \%$ fetal bovine serum (FBS; Invitrogen, Carlsbad, CA), and $10 \mu \mathrm{g} / \mathrm{ml}$ gentamicin (Sigma, St. Louis, $\mathrm{MO}$ ) at $37^{\circ} \mathrm{C}$ in $5 \% \mathrm{CO}_{2}$. Cells were passaged at 1:4 dilution with fresh medium once every 4 days. Trastuzumab, also known as Herceptin (Genentech Inc., San Francisco, CA), was obtained from The Ohio State University Hospital Pharmacy. The PDK-1 inhibitor OSU-03012 was synthesized as described previously (Zhu et al., 2004). Mouse monoclonal anti-poly(ADP-ribose) polymerase (PARP) was purchased from Cell Signaling Technology Inc. (Beverly, MA). Rabbit antibodies against HER2, phospho-Thr ${ }^{308}$-Akt, Akt, and p27 ${ }^{\text {kip } 1}$, and the mouse antibody against $\mathrm{p} 70^{S 6 K}$ were obtained from Santa Cruz Biotechnology, Inc. (Santa Cruz, CA). Rabbit anti-phospho-Thr ${ }^{229}-\mathrm{p} 70^{\text {S6K }}$ and anti-phospho-Thr ${ }^{157}$-p27 were from R\&D Systems Inc. (Minneapolis, MN). Mouse monoclonal antiactin was purchased from MP Biomedicals Inc. (Irvine, CA). Goat anti-rabbit and goat anti-mouse IgG-horseradish peroxidase conjugates were from Jackson ImmunoResearch Laboratories (West Grove, PA).

Cell Viability Assay. Cell viability was analyzed by the 3-(4,5dimethylthiazol-2-yl)-2,5-diphenyl-2H-tetrazolium bromide (MTT) assay (TCI America; Portland, OR) in six replicates. Cells (3000) were grown in 10\% FBS-supplemented DMEM/F12 medium in 96well, flat-bottomed plates and were exposed to various concentrations of individual agents or combination of drugs dissolved in DMSO (final concentration, $\leq 0.1 \%$ ) in the same medium. Control groups received DMSO vehicle at a concentration equal to that in drugtreated cells. After 72 -h treatment, medium was removed and replaced by $200 \mu \mathrm{l}$ of MTT $(0.5 \mathrm{mg} / \mathrm{ml})$ in DMEM/F12 medium, and cells were incubated in the $\mathrm{CO}_{2}$ incubator at $37^{\circ} \mathrm{C}$ for $3 \mathrm{~h}$. After discarding the MTT solution, the reduced MTT dye was solubilized with $200 \mu \mathrm{l} /$ well DMSO and was analyzed by measuring the absorbance at $570 \mathrm{~nm}$ in a plate reader. The $\mathrm{IC}_{50}$ value, which represents the drug concentration required for $50 \%$ growth inhibition, was calculated with the Calcusyn software (Biosoft, Cambridge, UK) by using the median-effect method (Chou and Talalay, 1984).

Immunoblotting. The general procedure for the Western blot analysis was performed as follows. Cells were scratched from culture flasks and collected by centrifugation at $2000 \mathrm{~g}$ and resuspended in RIPA lysis buffer consisting of $50 \mathrm{mM}$ Tris-HCl, $\mathrm{pH} 7.4,150 \mathrm{mM}$ $\mathrm{NaCl}, 1 \mathrm{mM}$ EDTA, $1 \%$ Triton X-100, 1\% sodium deoxycholate, $0.1 \%$ SDS, and a mixture of protease inhibitor cocktail (Calbiochem, La Jolla, CA) and phosphatase inhibitors ( $10 \mu \mathrm{M}$ sodium fluoride, $5 \mu \mathrm{M}$ sodium vanadate, and $10 \mu \mathrm{M} \beta$-glycerol phosphate). The mixture was sonicated for $5 \mathrm{~s}$, and protein contents were analyzed by using the Bradford assay kit (Bio-Rad, Hercules, CA). Fifty micrograms of total proteins was resolved in SDS-polyacrylamide gels on a Minigel apparatus and transferred to a nitrocellulose membrane using a semidry transfer cell. After blocking with Tris-buffered saline containing $0.05 \%$ Tween 20 (TBST) and 5\% nonfat milk for 60 min, the membrane was incubated with the appropriate primary antibody at 1:1000 dilution in TBST-1\% nonfat milk at $4^{\circ} \mathrm{C}$ overnight and washed three times with TBST. The membrane was probed with horseradish peroxidase-conjugated secondary antibodies at 1:3000 for $1 \mathrm{~h}$ at room temperature and washed with TBST three times. The immunoblots were visualized by enhanced chemiluminescence.

Western Blot Analysis of PARP Cleavage. Drug-treated cells for $72 \mathrm{~h}$ were collected, washed with ice-cold PBS, and resuspended in the aforementioned RIPA lysis buffer. Soluble cell lysates were collected after centrifugation at $10,000 \mathrm{~g}$ for $5 \mathrm{~min}$. Equivalent amounts of proteins $(50 \mu \mathrm{g})$ from each lysate were resolved in $10 \%$ SDS-polyacrylamide gels. Bands were transferred to nitrocellulose membranes and analyzed by immunoblotting.

Analysis of DNA Fragmentation by ELISA. Induction of apoptosis was assessed with a Cell Death Detection ELISA kit (Roche Diagnostics, Indianapolis, IN) following the manufacturer's instruction. This test is based on the quantitative determination of cytoplasmic histone-associated DNA fragments in the form of mononucleosomes and oligonucleosomes after induced apoptotic death. In brief, $1 \times 10^{6}$ cells were exposed to individual treatments in a T-25 flask in 10\% FBS-supplemented DMEM/F12 medium for $72 \mathrm{~h}$. Both 
floating and adherent cells were collected; cell lysates equivalent to $5 \times 10^{5}$ cells were used in the ELISA.

Flow Cytometric Analysis. Fluorescein-conjugated annexin V and propidium iodide (PI) were used to quantify the percentage of cells undergoing apoptosis by following the protocol by the manufacturer (Molecular Probes, Eugene, OR). In short, after exposure to trastuzumab with or without OSU-03012 in 10\% FBS-supplemented DMEM/F12 medium for $24 \mathrm{~h}$, cells were collected and resuspended in $1 \mathrm{ml}$ of binding buffer (10 mM HEPES, $\mathrm{pH} 7.4,140 \mathrm{mM} \mathrm{NaCl}$, and 2.5 $\mathrm{mM} \mathrm{CaCl}{ }_{2}$ ) at a concentration of $5 \times 10^{6}$ cells $/ \mathrm{ml}$. A $200-\mu \mathrm{l}$ solution was transferred to a culture tube, to which were added fluoresceinconjugated annexin V and PI. The cells were gently vortexed and incubated for $15 \mathrm{~min}$ at room temperature in the dark. An additional $800 \mu \mathrm{l}$ of binding buffer was added to each tube, and the samples were analyzed by a fluorescence-activated cell sorter.

Immunocytochemical Analysis. SKBR and SKBR3/IGF-IR cells were treated with $5 \mu \mathrm{M}$ OSU-03012 in 10\% FBS-supplemented DMEM/F12 medium for $24 \mathrm{~h}$, washed with Dulbecco's PBS, fixed with $4 \%$ paraformaldehyde for $30 \mathrm{~min}$ at room temperature, and then washed with PBS. The fixed cells were permeabilized with $0.1 \%$ Triton X-100 in 1\% FBS-containing PBS, stained with HER2 antibodies at 1:100 dilution (Santa Cruz Biotechnology) for $24 \mathrm{~h}$ at $4^{\circ} \mathrm{C}$, and treated with Alexa Fluor 647 goat anti-rabbit IgG (Molecular Probes) in the aforementioned PBS for conjugation. The nuclear counter staining was performed using a 4,6-diamidino-2-phenylindole-containing mounting medium (Vector Laboratories, Burlingame, CA) before examination. Images of immunocytochemically labeled samples were observed using a Zeiss confocal microscope (LSM510; Carl Zeiss Inc., Thornwood, NY) with an argon laser and a helium-neon laser and appropriate filters (excitation wavelength, $633 \mathrm{~nm}$ for HER2 and $543 \mathrm{~nm}$ for 4,6-diamidino-2-phenylindole).

Statistical Analysis. Each experiment was performed in triplicate. All experiments were carried out at least two times on different occasions. The medium-effect method was used to analyze doseresponse data for single or multiple drugs. The synergistic effect of multiple drugs was determined by the definition of Chou and Talalay (1984). Cells were treated with series dilution of trastuzumab and OSU-03012 at fixed concentration ratios. The concentration of trastuzumab ranged from 1 to $10 \mu \mathrm{g} / \mathrm{ml}$, whereas that of OSU-03012 was 0.5 to $5 \mu \mathrm{M}$. These two drugs were assumed as totally independent modes of action and were therefore mutually nonexclusive. By using the software package Calcusyn (Biosoft, Cambridge, UK), the values of combination index (CI) were calculated. The values of CI at different levels of growth inhibition were calculated based on the formula for mutually nonexclusive mechanism: $\left(\mathrm{D}_{1} / \mathrm{D}_{\mathrm{x} 1}\right)+\left(\mathrm{D}_{2} / \mathrm{D}_{\mathrm{x} 2}\right)+$ $\left(\mathrm{D}_{1} \mathrm{D}_{2} / \mathrm{D}_{\mathrm{xl}} \mathrm{D}_{\mathrm{x} 2}\right)$, where $\mathrm{D}_{\mathrm{x} 1}$ and $\mathrm{D}_{\mathrm{x} 2}$ are the doses of drug 1 and drug 2 alone required to produce $x$ percentage effect, and $D_{1}$ and $D_{2}$ are the doses of drug 1 and drug 2 in combination required to produce the same effect. The CI values less than 1 , equal to 1 , and greater than 1 are indicative of synergism, additive effect, and antagonism, respectively.

\section{Results}

Differential Sensitivity of MDA-MB-231, BT-474, SKBR3, and SKBR3/IGF-IR Cells to Trastuzumab. These four different breast cancer cell lines exhibited distinct molecular characteristics, which underlie differences in the respective sensitivity to trastuzumab's antiproliferative effects (Fig. 1). Among them, MDA-MB-231 cells lacked HER2 expression, whereas BT474, SKBR3, and SKBR3/IGF-IR cells displayed high abundance of HER2 (Fig. 1A). Moreover, SKBR3/IGF-IR cells showed ectopic IGF-IR overexpression. These four cell lines exhibited different levels of Akt phosphorylation, in the order of SKBR3/IGF-IR > BT474, SKBR3 $\gg$ MDA-MB-231.

Among these four cell lines, MDA-MB-231 cells were in- sensitive to trastuzumab because of lack of HER2 expression. Exposure of HER2-overexpressing BT474 and SKBR3 cells to trastuzumab caused a dose-dependent decrease in cell proliferation, which leveled off at the maximum cell reduction of approximately 50\% (Fig. 1B, left). However, SKBR3/IGF-IR cells acquired a resistant phenotype as a result of IGF-IR overexpression (Lu et al., 2001). Despite the discrepancy in trastuzumab sensitivity, these four cell lines were equally susceptible to the antiproliferative effect of OSU-03012, indicating a lack of cross-resistance to OSU-03012 in trastuzumab-resistant SKBR3/IGF-IR cells (Fig. 1B, right). The respective $\mathrm{IC}_{50}$ values were the following: MDA-MB-231, $3.8 \pm 0.4 \mu \mathrm{M}$; BT474, $3.9 \pm 0.4 \mu \mathrm{M}$; SKBR3, $3.0 \pm 0.2 \mu \mathrm{M}$; and SKBR3/IGF-IR, $3.2 \pm 0.1 \mu \mathrm{M}$.

To shed light onto the mechanism responsible for this differential response, we examined the effects of trastuzumab and OSU-03012 on the expression and/or phosphorylation status of a number of pertinent signaling markers in individual cell lines. These biomarkers included HER2, Akt, the CDK inhibitor $\mathrm{p} 27^{k i p 1}$, and the PDK1 substrate $\mathrm{p} 70^{\mathrm{S} 6 K}$. $\mathrm{p} 27^{\text {kip } 1}$ is known to be phosphorylated by Akt, which impairs the nuclear import and action of this CDK inhibitor (Liang et al., 2002). In BT474 and SKBR3 cells, trastuzumab caused a dose-dependent decrease in HER2 expression, accompanied by increased $\mathrm{p} 27^{k i p 1}$ expression and decreased phosphorylation of Akt, p27 $7^{k i p 1}$, and, to a lesser extent, $\mathrm{p} 70^{\mathrm{S} 6 \mathrm{~K}}$ (Fig. 2). None of these effects, however, was noted in low HER2expressing MDA-MB-231 or trastuzumab-resistant SKBR3/ IGF-IR cells within the dose range examined, underlying the lack of antiproliferative effects of trastuzumab in these two cell lines.
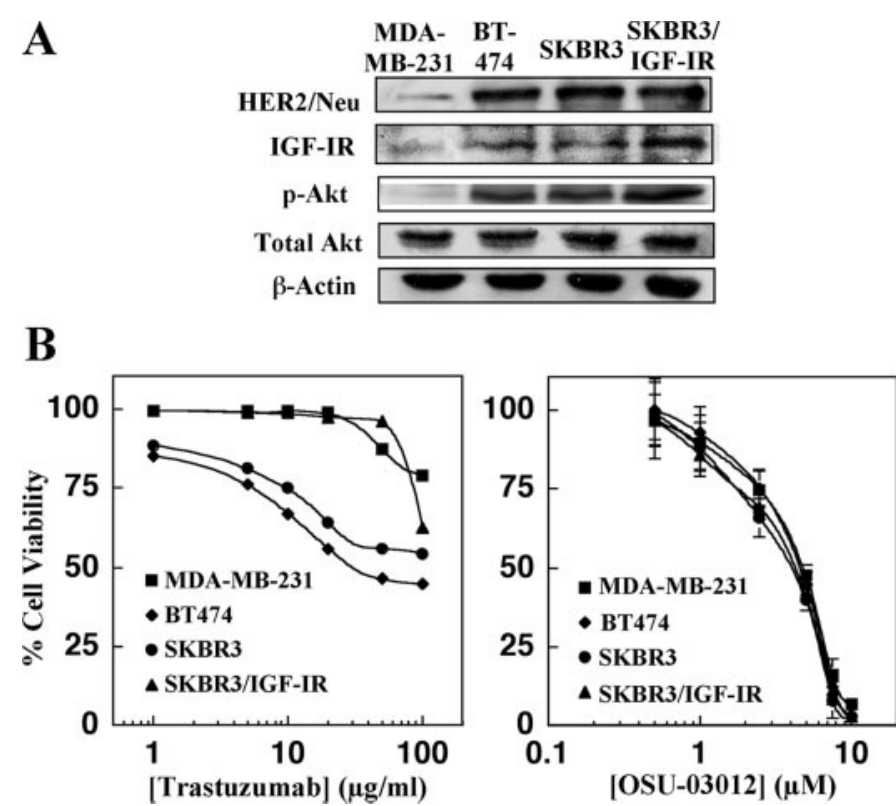

Fig. 1. MDA-MB-231, BT474, SKBR3, and SKBR3/IGF-IR cells exhibit differential susceptibility to trastuzumab but are equally sensitive to OSU-03012. A, status of HER2 expression, IGF-IR expression, and $\mathrm{Thr}^{308}$-Akt phosphorylation in these four cell lines. As shown, Akt phosphorylation is up-regulated in BT474, SKBR3, and SKBR3-IGF-IR cells, which is associated with the overexpression of HER2 and IGF-IR. These immunoblots are representatives of three independent experiments. B, dose-response curves obtained by MTT assay after 72-h exposure of these four cell lines to trastuzumab (left) and OSU-03012 (right). Each data point represents means \pm S.D. $(n=6)$. 
OSU-03012 Induces Apoptosis through PDK-1 Inhibition Irrespective of HER2 Expression Levels and Resistant Phenotype. OSU-03012 was able to trigger apoptosis in all four cell lines at low micromolar concentrations irrespective of the HER2 expression status and resistant phenotype, as evidenced by PARP cleavage (Fig. 2). As shown, exposure of these four different types of cells to OSU03012 caused dose-dependent decreases in the phosphorylation levels of Akt, $\mathrm{p} 27^{k i p 1}$, and $\mathrm{p} 70^{\mathrm{S} 6 K}$. Moreover, the extent of dephosphorylation in these biomarkers in trastuzumabinsensitive MDA-MB-231 and SKBR3/IGF-IR cells was in line with that of BT474 and SKBR3 cells, suggesting that this apoptosis induction was, at least in part, attributable to the ability of OSU-03012 to down-regulate PDK-1/Akt signaling. In contrast to trastuzumab, OSU-03012 has no effect on p27 $7^{k i p 1}$ expression. Nevertheless, this PDK-1 inhibitor was able to repress HER2 expression in a manner similar to that of trastuzumab (Fig. 2). This OSU-03012-mediated HER2 down-regulation was reminiscent of the effect of the Hsp90 inhibitor geldanamycin and its analogs on HER2 degradation (Zheng et al., 2000). Immunocytochemical analysis indicates that OSU-03012 decreased the cell surface expression of HER2 and facilitated the internalization of the receptor in both SKBR3 and SKBR/IGF-IR cells, which was also noted with the PI3K inhibitor LY294002 (Fig. 3). This finding

suggests a link between receptor internalization and HER2 down-regulation. The underlying mechanism is currently under investigation.

OSU-03012 Restores the Sensitivity of SKBR/IGF-IR Cells to Trastuzumab's Antiproliferative Effect. In light of the important role of Akt in the antitumor action of trastuzumab, we hypothesized that concurrent inhibition of PDK-1/Akt signaling by OSU-03012 could sensitize trastuzumab-resistant cells to the residual antiproliferative activity of this antibody. To test this hypothesis, MDA-MB-231, BT474, SKBR3, and SKBR3/IGF-IR cells were exposed to varying concentrations of trastuzumab in the presence of 2.5 or $5 \mu \mathrm{M}$ OSU-03012 versus DMSO control for $72 \mathrm{~h}$. Figure 4 demonstrates the ability of OSU-03012 to improve the sensitivity of HER2-overexpressing cells to trastuzumab-induced cell death. For example, $10 \mu \mathrm{g} / \mathrm{ml}$ trastuzumab and 2.5 $\mu \mathrm{M}$ OSU-03012 alone caused approximately 25 and $30 \%$, respectively, cell reduction in BT474 and SKBR3 cells, and the combination of these two agents could achieve $65 \%$ cell death. Especially noteworthy, OSU-03012 was able to overcome the resistant phenotype in SKBR3/IGF-IR cells. Although trastuzumab alone at concentrations lower than 50 $\mu \mathrm{g} / \mathrm{ml}$ was ineffective in inhibiting SKBR3/IGF-IR cell proliferation, OSU-03012 restored the sensitivity of these resistant cells to trastuzumab's antiproliferative effects to an

BT474

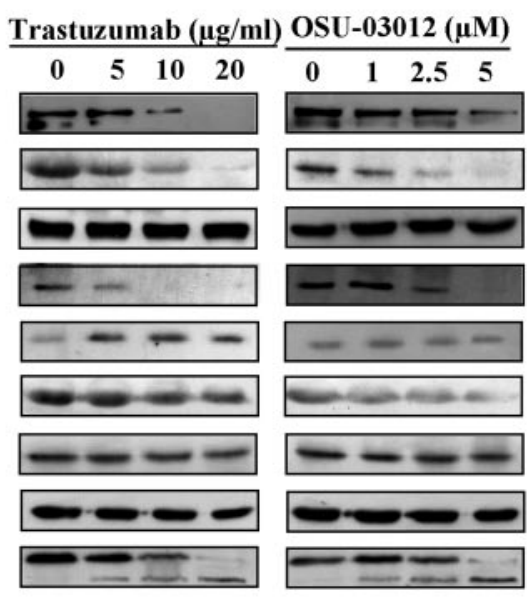

\section{SKBR3}

Trastuzumab $(\mu \mathrm{g} / \mathrm{ml}) \quad$ OSU-03012 $(\mu \mathrm{M})$

HER2/Neu Trastuzumab $(\mu \mathrm{g} / \mathrm{ml})$ OSU-03012 $(\mu \mathrm{M})$ $\begin{array}{lllll}0 & 5 & 10 & 20\end{array}$

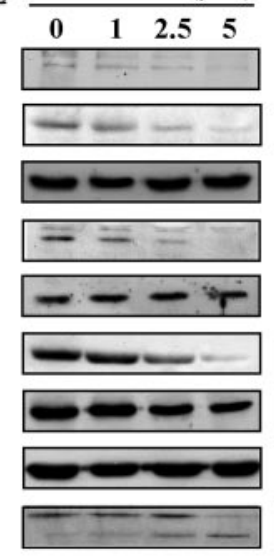

PARP

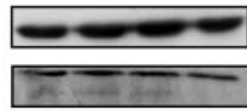

\section{SKBR3/IGF-IR}

Trastuzumab $(\mu \mathrm{g} / \mathrm{ml})$ OSU-03012 ( $\mu \mathrm{M})$
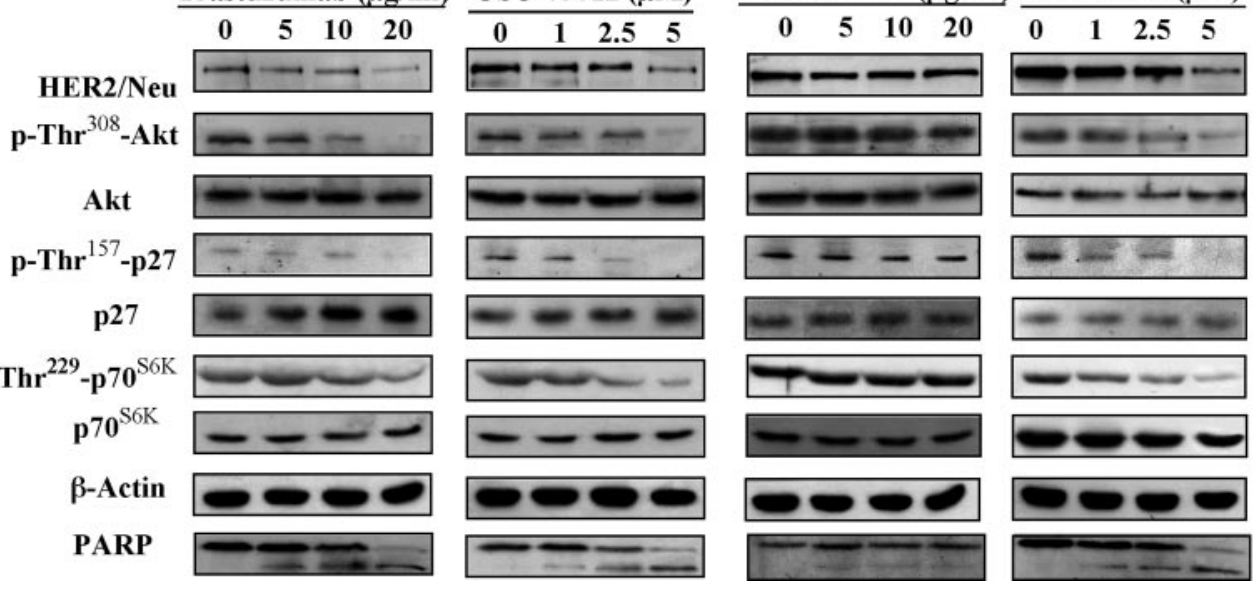

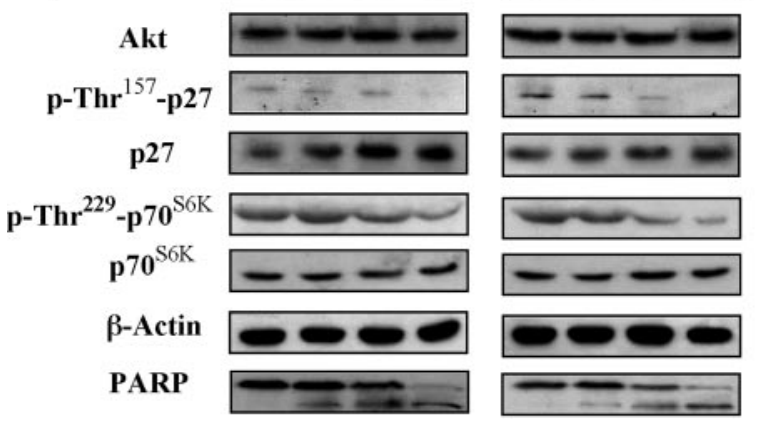

Fig. 2. Dose-dependent effect of trastuzumab and OSU-03012 on the expression and/or phosphorylation levels of HER2, Akt, p27 $7^{k i p 1}$, and $\mathrm{p} 70^{\text {S6K }}$, and PARP cleavage in MDA-MB-231, BT474, SKBR3, and SKBR3/IGF-IR cells after 24-h exposure. These immunoblots are representatives of three independent experiments. 
extent similar to that of the parent cell line SKBR3 (Fig. 4A). This sensitizing effect, however, was not noted in the low HER2-expressing MDA-MB-231 cells.

We used SKBR3 and SKBR3/IGF-IR cells to obtain evidence that the chemosensitizing effect of OSU-03012 was attributable to its ability to enhance the susceptibility of these HER2overexpressing cells to trastuzumab-induced apoptosis. DNA fragmentation ELISA indicates that trastuzumab alone did not cause appreciable increase in nucleosome formation even at $20 \mu \mathrm{g} / \mathrm{ml}$. However, in the presence of $5 \mu \mathrm{M}$ OSU-03012, trastuzumab significantly increased the extent of DNA fragmentation in a dose-dependent manner ( $*, P<0.005$ compared with $5 \mu \mathrm{M}$ OSU-03012 alone) (Fig. 5A). The effect of $5 \mu \mathrm{M}$ OSU-03012 on sensitizing both cell lines to trastuzumab-induced apoptosis was also confirmed by the annexin V/PI staining (Fig. 5B). As shown, $10 \mu \mathrm{g} / \mathrm{ml}$ trastuzumab and $5 \mu \mathrm{M}$ OSU-03012 alone gave rise to 12 and $35 \%$, respectively, apoptosis compared with $13 \%$ for the vehicle control in SKBR3 cells (top). In contrast, treatment of SKBR3 cells with

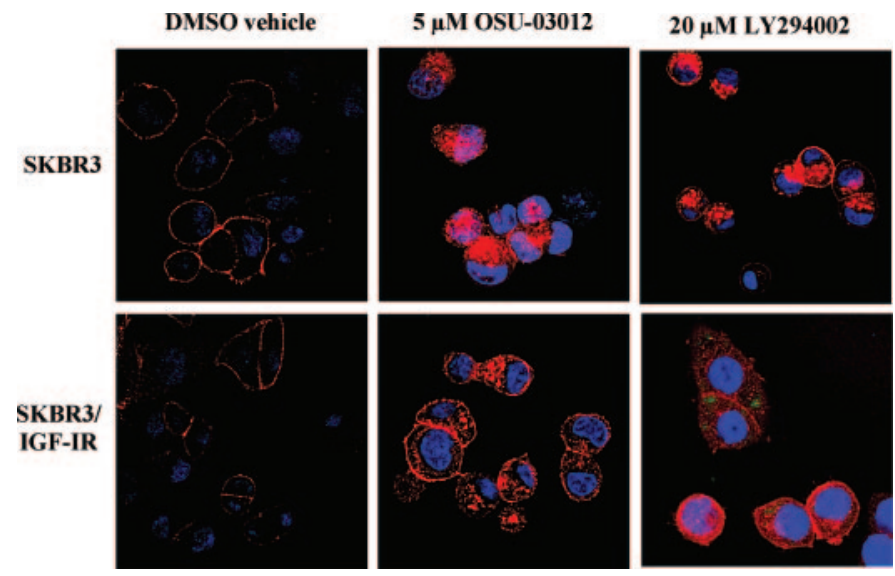

Fig. 3. Immunocytochemical examination of HER2 localization in SKBR3 and SKBR3/IGF-IR cells treated with DMSO vehicle, $5 \mu \mathrm{M}$ OSU-03012, and $20 \mu \mathrm{M}$ LY294002 for $24 \mathrm{~h}$. a combination of 5 and $10 \mu \mathrm{g} / \mathrm{ml}$ trastuzumab with $5 \mu \mathrm{M}$ OSU-03012 led to 60 and 89\%, respectively, apoptotic death. A similar finding was also noted with the IGF-IR-overexpressing SKBR3 cells (bottom).

To demonstrate that there existed synergism between these two drugs, we carried out the medium dose analysis of the combined effect of OSU-03012 and trastuzumab at a fixed ratio on cell viability according to the median-effect method (Chou and Talalay, 1984). Figure 6A depicts the dose-response curves of these four cell lines after 72-h treatment of five different dose combinations of trastuzumab (nanomolar)/ OSU-03012 (micromolar) at a fixed ratio (7/0.5, 14/1, 28/2, $42 / 3,56 / 4$, and $70 / 5$; $\bigcirc)$ versus varying concentrations of trastuzumab $(\bigcirc)$ and OSU-03012 $(\triangle)$ alone. These dose-response data were used to determine CI values in relation to the fraction affected (Fig. 6B). With the exception of MDAMB-231, the other three cell lines exhibited CI values all lower than 1, which was indicative of synergism between trastuzumab and OSU-03012 in HER2-overexpressing cells. For example, the CI values calculated for the combination treatment that caused $50 \%$ cell death (fractional effect, 0.5 ) were $0.96,0.50,0.71$, and 0.68 for MDA-MB-231, BT474, SKBR3, and SKBR3/IGF-IR cells, respectively.

OSU-03012 Overcomes Trastuzumab Resistance in SKBR3/IGF-IR Cells by Antagonizing the Protective Effect of IGF-IR Overexpression on Trastuzumab-Mediated HER2 Repression, Akt Dephosphorylation, and p27 ${ }^{\text {Kip1 }}$ Up-Regulation. From a mechanistic perspective, OSU-03102-mediated sensitization of SKBR3/IGF-IR cells to trastuzumab could be accounted for by the ability of this PDK-1/Akt signaling inhibitor to counter the protective effect of IGF-IR overexpression on critical aspects of signal transduction pertinent to trastuzumab's antiproliferative activity, including HER2 expression, Akt phosphorylation, and p2 $7^{\text {kip } 1}$ phosphorylation and expression (Fig. 7). In all three HER2-overexpressing cell lines, the extent of changes in these biomarkers by the combination treatment seemed to be
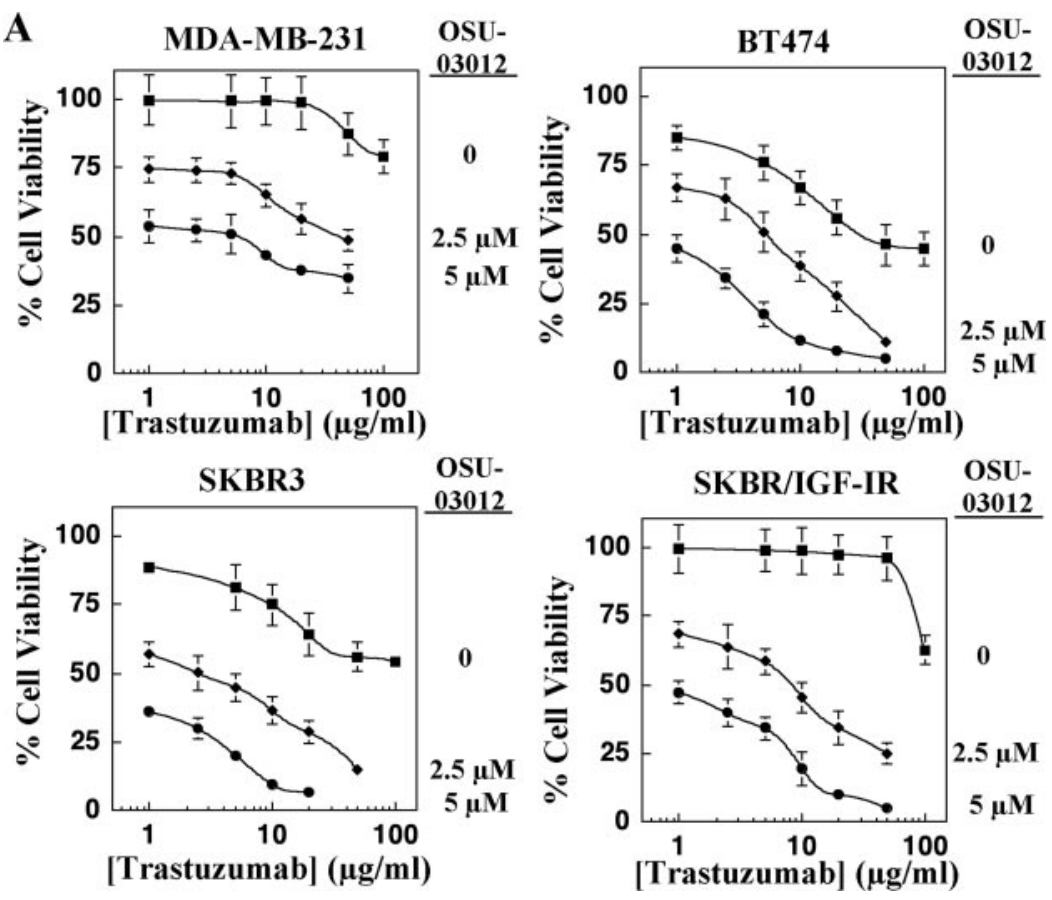

Fig. 4. OSU-03012 sensitizes BT474, SKBR3, and SKBR3/ IGF-IR cells but not MDA-MB-231 cells to trastuzumab's antiproliferative effects. A, dose-response curves were obtained by MTT assays after 72-h exposure of these four cell lines to a combination of 2.5 or $5 \mu \mathrm{M}$ OSU-03012 and varying concentrations of trastuzumab. Each data point represents means \pm S.D. $(n=6)$.

03012 
greater than the addition of individual effects. For example, trastuzumab alone, within the dose range of 5 to $20 \mu \mathrm{g} / \mathrm{ml}$, had very little, if any, effects on any of these biomarkers in SKBR3/IGF-IR cells. However, it could facilitate the effect of OSU-03012 on HER2 degradation, Akt dephosphorylation, and $\mathrm{p} 27^{\text {kip } 1}$ expression and dephosphorylation in a dosedependent manner. In line with the cell viability data, this mechanistic synergy was not noted in MDA-MB-231 cells.

\section{Discussion}

Although trastuzumab is useful in treating patients with HER2-overexpressing metastatic breast cancer, overcoming trastuzumab resistance represents an important challenge to improve patient outcomes. Although the mechanisms underlying the acquisition of trastuzumab resistance are not yet well-defined, recent evidence suggests that HER2-positive cells might develop trastuzumab resistance by raising the threshold against the antibody's antiproliferative effect through the up-regulation of PI3K/Akt signaling or downregulation of the CDK inhibitor $\mathrm{p} 27^{k i p 1}$. Thus, combination of trastuzumab with a PI3K/Akt signaling inhibitor represents a viable strategy to address this resistance issue (Pandolfi, 2004).

The present study indicates that concurrent inhibition of PDK-1 with OSU-03012 reverses the trastuzumab-resistant phenotype in IGF-IR-overexpressing SKBR3 cells. These trastuzumab-resistant cells exhibited the same susceptibility to OSU-03012 as their parent (SKBR3) cells as well as BT474 and MDA-MB-231 cells irrespective of the resistance status. The lack of cross-resistance underscores the functional relevance of targeting PDK-1/Akt signaling in trastuzumab-resistant cells. Despite the resistant phenotype, trastuzumab still retains its ability to bind HER2 and to activate downstream apoptotic signaling in resistant cells. Therefore, OSU03012 could sensitize trastuzumab-resistant cells to trastuzumab's antitumor action through PDK-1 inhibition, as evidenced by the dephosphorylation of Akt, p27 $7^{\text {kip } 1}$, and $\mathrm{p} 70^{\mathrm{S} 6 K}$. It is noteworthy that Western blot analysis indicates that OSU-03012 caused the repression of HER2 expression in a manner similar to that of trastuzumab. Immunocytochemical analysis shows that OSU-03012 treatment led to HER2 internalization in both SKBR3 and SKBR3/IGF-IR cells, which presumably led to receptor degradation. The effect of
B

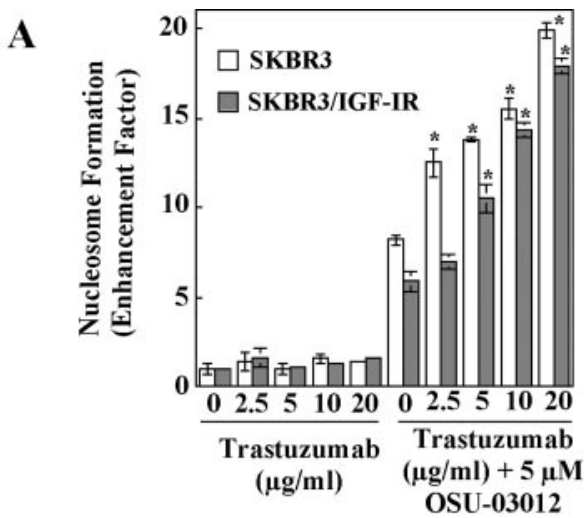

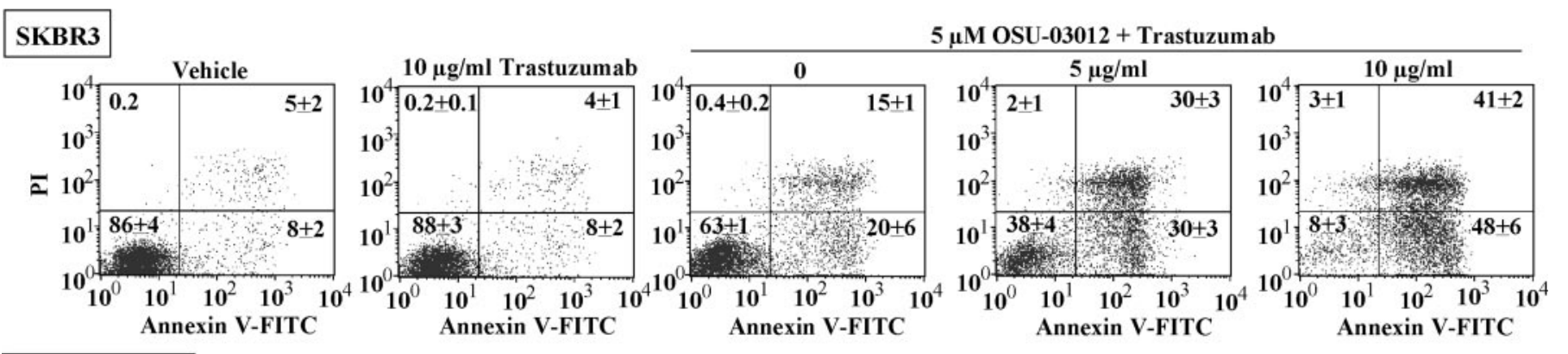

$5 \mu \mathrm{M}$ OSU-03012 + Trastuzumab

\section{SKBR3-IGF1R}

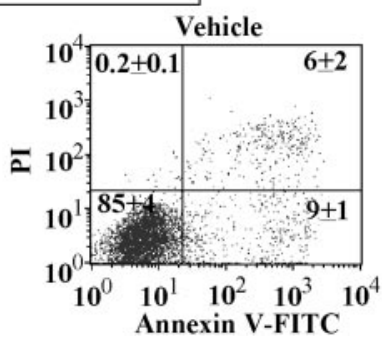

$5 \mu \mathrm{M}$ OSU-03012 + Trastuzumab
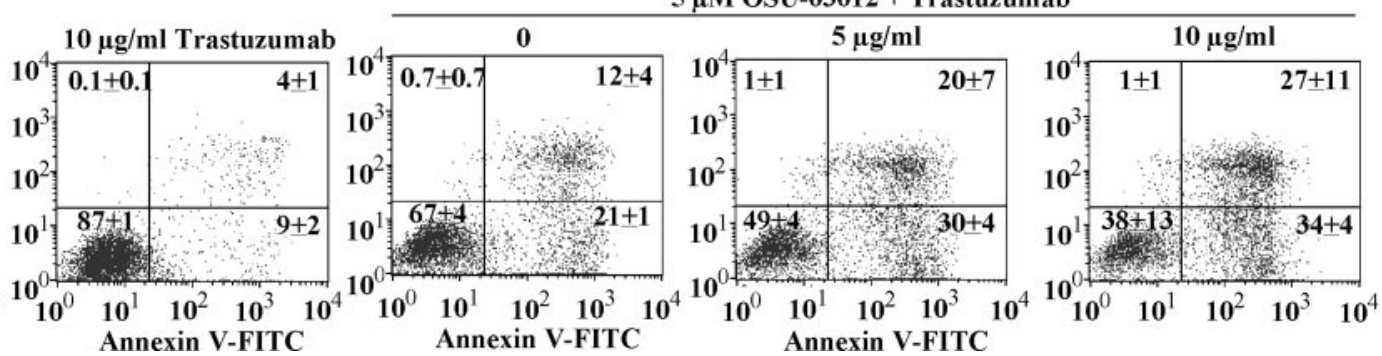

Fig. 5. Evidence that the ability of OSU-03012 to sensitize SKBR3 and SKBR3/IGF-IR breast cancer cells to trastuzumab's antiproliferative effect was due to apoptosis induction. A, quantitative measurement of the formation of nucleosomes by a cell death detection ELISA kit after 72-h exposure to individual treatments. Columns, mean; bars, S.D. $(n=3)$. B, flow cytometric analysis of apoptotic death in SKBR3 (top) and SKBR3/IGF-IR (bottom) cells after 24-h exposure to individual treatments. Results are representative of three independent experiments. The percentages in the graphs represent the percentage of cell numbers in each quadrant. 
OSU-03012 on HER2 down-regulation is reminiscent of that of the Hsp90 inhibitor geldanamycin on HER-family tyrosine kinases((Zheng et al., 2000), although the underlying mechanisms might differ. It was speculated that geldanamycin might repress the expression of HER kinases through the disruption of the interaction between $\mathrm{Hsp} 90$ and the catalytic domain of HER kinases (Zheng et al., 2000). We rationale that the effect of OSU-03012 on HER2 down-regulation might be attributable to the inhibition of PDK-1/Akt signaling because LY294002 could also achieve a similar effect. OSU-03012's ability to suppress HER2 expression is noteworthy because HER2 degradation represents a major mechanism underlying the antiproliferative effect of trastuzumab and other anti-HER2 antibodies (Hurwitz et al., 1995; Frankel, 2002). A correlation has been shown between the effectiveness in HER2 down-regulation and the in vivo potency of different anti-HER2 antibodies in tumor growth inhibition (Harwerth et al., 1993). Reduced HER2 at the cell surface allows less HER2 heterodimer formation, resulting in reduced growth factor-induced signaling and proliferation.

The combination of trastuzumab and an epidermal growth factor receptor inhibitor such as gefitinib (Iressa; AstraZeneca Pharmaceuticals LP, Wilmington, DE) or erlotinib (Tarceva; OSI Pharmaceuticals, Melville, NY) is being evaluated as a strategy to prevent or delay resistance in HER2positive patients (Miller, 2004). The ability of gefitinib to enhance the efficacy of trastuzumab has been demonstrated in several sensitive breast cancer cell systems (Moulder et al., 2001). However, in a phase I trial in patients with pretreated metastatic breast cancer, the combination of trastuzumab and gefitinib was well tolerated but showed poor antitumor activity.

Because PDK-1/Akt signaling represents important downstream effectors of many receptor tyrosine kinases, use of the PDK-1 inhibitor OSU-03012 enhances the efficacy in sensitive cells, and, more importantly, restores sensitivity in resistant cells. It is noteworthy that OSU-03012 is currently undergoing preclinical testing under the Rapid Access to Intervention Development program at the National Cancer Institute. Our data indicate that oral administration of this agent in tumor-bearing nude mice at $200 \mathrm{mg} / \mathrm{kg}$ for 1 month achieves a peak serum concentration of more than $20 \mu \mathrm{M}$

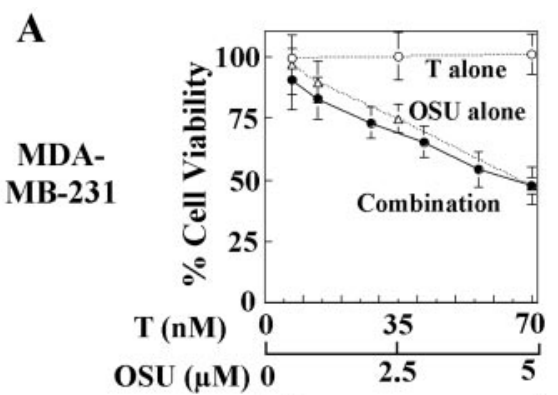

B

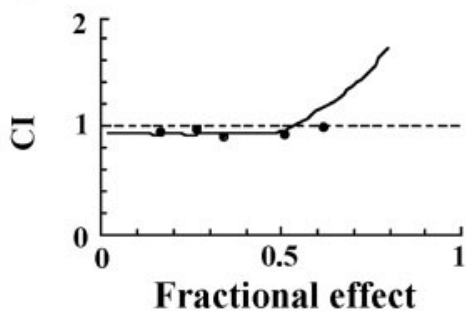

Fractional effect
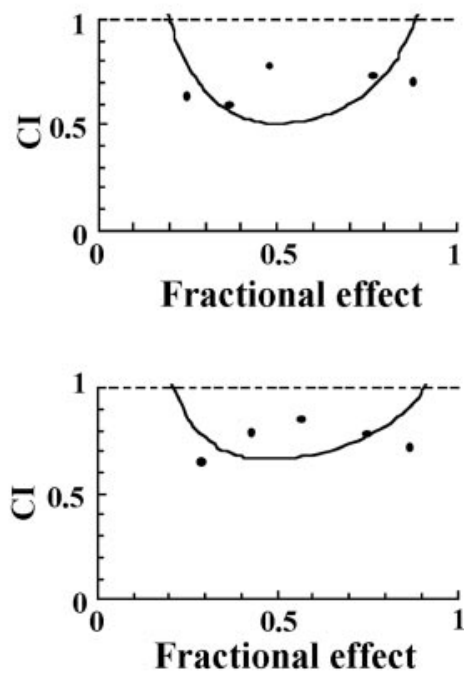

Fig. 6. Synergistic effect of OSU-03012 on sensitizing HER2-overexpressing cells to trastuzumab. A, MDA-MB231, BT474, SKBR3, and SKBR/IGF-IR cells were exposed to six dose combinations of trastuzumab (nanomolar)/OSU03012 (micromolar) at a fixed ratio, including $7 / 0.5,14 / 1$, $28 / 2,42 / 3,56 / 4$, and 70/5. The concentrations of $7,14,28$ 42,56 , and $70 \mu \mathrm{M}$ trastuzumab were equivalent to $1,2,4$, 6,8 , and $10 \mu \mathrm{g} / \mathrm{ml}$ of the antibody. Cell viability was analyzed by MTT after $72 \mathrm{~h}$ treatment $(\bullet)$. Broken lines represent the dose-response curves of trastuzumab $(O)$ and OSU-03012 $(\triangle)$ alone in the respective cell lines. B, CI values for cell death were determined in relation to the fraction affected using the medium dose analysis. CI values of less than 1 are considered as a synergistic interaction. Mutually nonexclusive $\mathrm{CI}$ for combination at the $\mathrm{IC}_{50}$ value was 0.962 for MDA-MB-231, 0.501 for BT474, 0.712 for SKBR3, and 0.677 for SKBR3/IGF-IR. The curves depict the simulations of mutually nonexclusive CI values by Calcusyn.
SKBR3
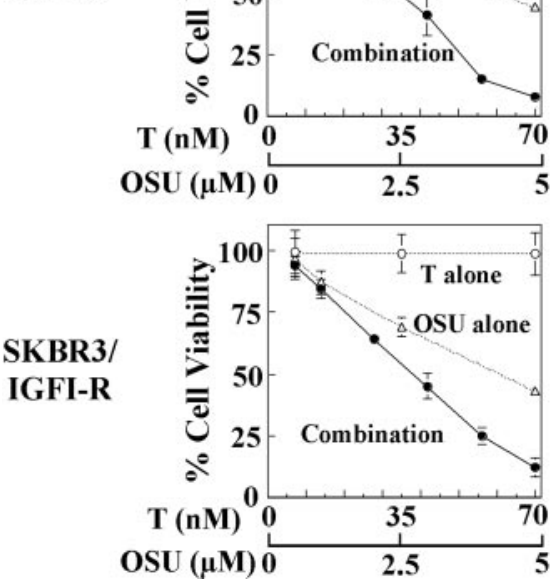

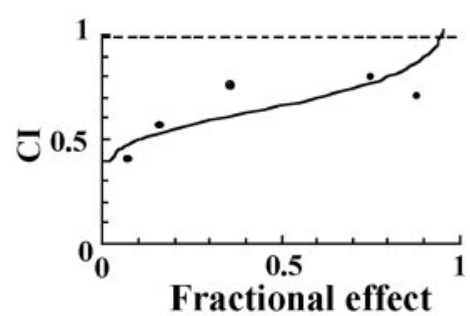




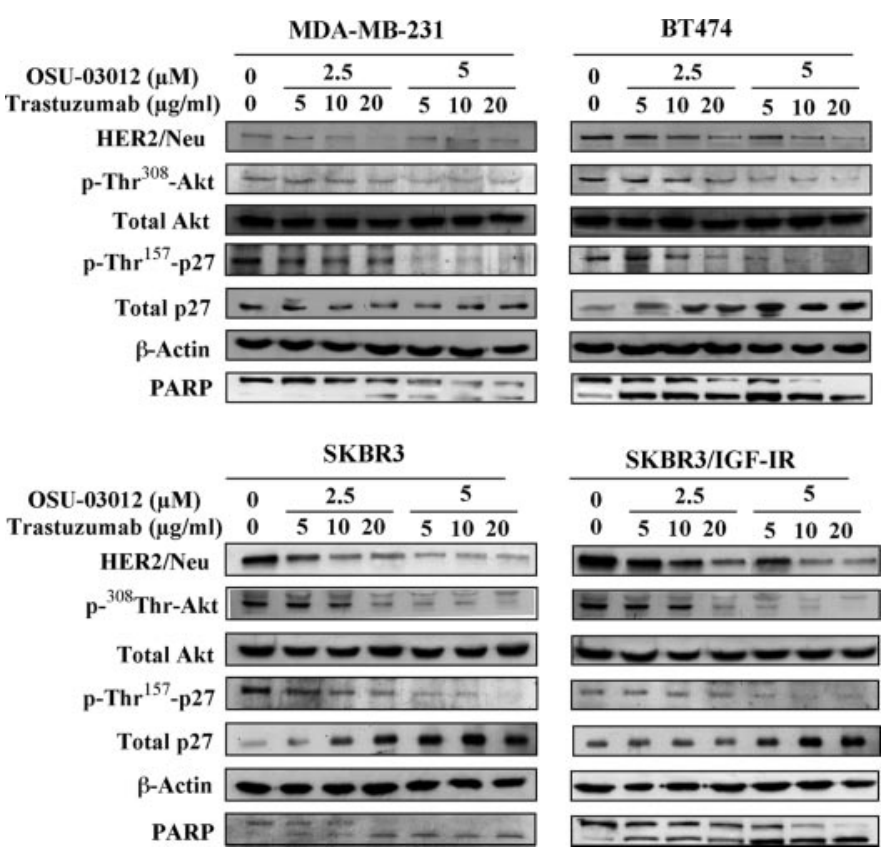

Fig. 7. Combination effect of OSU-03012 and trastuzumab at different concentrations on the expression/phosphorylation level of HER2, Akt and $\mathrm{p} 27^{\text {kip } 1}$, and PARP cleavage in MDA-MB-231, BT474, SKBR3, and SKBR3/IGF-IR cells after 24-h exposure. These immunoblots are representatives of three independent experiments.

with no apparent toxicity at necropsy (S.K. Kulp and C.-S. Chen, unpublished data). Our data indicate that low doses of OSU-03012 were able to synergize with trastuzumab in inducing apoptotic death in HER2-positive cells.

The potential clinical relevance of the trastuzumab-OSU03012 combination is considerable. Not only do our data suggest that the trastuzumab-OSU-03012 combination represents a novel strategy to overcome trastuzumab resistance, but the in vitro evidence also suggests that OSU-03012 can increase the efficacy of trastuzumab in HER2-positive breast cancer treatment. The regimen of weekly trastuzumab dosing at $2 \mathrm{mg} / \mathrm{kg}$ or every 3 -week dosing at $6 \mathrm{mg} / \mathrm{kg}$ will achieve a steady-state serum level of 60 to $80 \mu \mathrm{g} / \mathrm{ml}$ (Leyland-Jones et al., 2003). In vitro cell viability assays using BT474 and SKBR3 cells indicate that the maximum cell reduction attainable at this range was approximately 50\%. However, trastuzumab in this combination range in combination with OSU-03012 at $2.5 \mu \mathrm{M}$ achieved greater than $90 \%$ killing.

Recent data provide evidence that development of resistance to trastuzumab in vivo is often associated with increased IGF-I receptor level (Cohen and Jani, 2005). This suggests that the SKBR3/IGFIR cell line we used may be a relevant model for at least a subset of spontaneously arising trastuzumab-resistant breast cancers. We are currently evaluating OSU-03012 in combination with trastuzumab in preclinical in vivo animal models. If the results of in vivo testing are promising, then this combination may represent a viable strategy for clinical testing in both trastuzumab-sensitive and -resistant HER2-positive breast cancer.

\section{References}

Arteaga CL (2003) Trastuzumab, an appropriate first-line single-agent therapy for HER2-overexpressing metastatic breast cancer. Breast Cancer Res 5:96-100.

Chou TC and Talalay P (1984) Quantitative analysis of dose-effect relationships: the combined effects of multiple drugs or enzyme inhibitors. Adv Enzyme Regul 22: 27-55.

Clark AS, West K, Streicher S, and Dennis PA (2002) Constitutive and inducible Akt activity promotes resistance to chemotherapy, trastuzumab, or tamoxifen in breast cancer cells. Mol Cancer Ther 1:707-717.

Cohen BD and Jani J (2005) Targeting the IGF1R signaling pathway: clinical implications. Proc Annu Meet Am Assoc Cancer Res 46:1459.

Crowder RJ and Ellis MJ (2005) Treating breast cancer through novel inhibitors of the phosphatidylinositol 3'-kinase pathway. Breast Cancer Res 7:212-214.

Frankel AE (2002) New HER2-directed therapies for breast cancer. Commentary re C. I. Spiridon et al., Targeting multiple her-2 epitopes with monoclonal antibodies results in improved antigrowth activity. Clin. Cancer Res., 8: 1720-1730, 2002 Clin Cancer Res 8:1699-1701.

Harwerth IM, Wels W, Schlegel J, Muller M, and Hynes NE (1993) Monoclonal antibodies directed to the erbB-2 receptor inhibit in vivo tumour cell growth. $\mathrm{Br} J$ Cancer 68:1140-1145.

Hurwitz E, Stancovski I, Sela M, and Yarden Y (1995) Suppression and promotion of tumor growth by monoclonal antibodies to ErbB-2 differentially correlate with cellular uptake. Proc Natl Acad Sci USA 92:3353-3357.

Hynes NE and Lane HA (2005) ERBB receptors and cancer: the complexity of targeted inhibitors. Nat Rev Cancer 5:341-354.

Kucab JE, Lee C, Chen CS, Zhu J, Gilks CB, Cheang M, Huntsman D, Yorida E Emerman J, Rollak M, et al. (2005) Celecoxib analogues disrupt Akt signaling, which is commonly activated in primary breast tumors. Breast Cancer Res 7:R796-R807.

Leyland-Jones B, Gelmon K, Ayoub JP, Arnold A, Verma S, Dias R, and Ghahramani $\mathrm{P}$ (2003) Pharmacokinetics, safety, and efficacy of trastuzumab administered every three weeks in combination with paclitaxel. J Clin Oncol 21:3965-3971.

Liang J, Zubovitz J, Petrocelli T, Kotchetkov R, Connor MK, Han K, Lee JH, Ciarallo $\mathrm{S}$, Catzavelos C, Beniston R, et al. (2002) PKB/Akt phosphorylates p27, impairs nuclear import of p27 and opposes p27-mediated G1 arrest. Nat Med 8:1153-1160. Lu Y, Zi X, Zhao Y, Mascarenhas D, and Pollak M (2001) Insulin-like growth factor-I receptor signaling and resistance to trastuzumab (Herceptin). J Natl Cancer Inst 93:1852-1857.

Miller KD (2004) The role of ErbB inhibitors in trastuzumab resistance. Oncologist 9 (Suppl 3): 16-19.

Moulder SL, Yakes FM, Muthuswamy SK, Bianco R, Simpson JF, and Arteaga CL (2001) Epidermal growth factor receptor (HER1) tyrosine kinase inhibitor ZD1839 (Iressa) inhibits HER2/neu (erbB2)-overexpressing breast cancer cells in vitro and in vivo. Cancer Res 61:8887-8895.

Nagata Y, Lan KH, Zhou X, Tan M, Esteva FJ, Sahin AA, Klos KS, Li P, Monia BP Nguyen NT, et al. (2004) PTEN activation contributes to tumor inhibition by trastuzumab, and loss of PTEN predicts trastuzumab resistance in patients. Cancer Cell 6:117-127.

Nahta R, Takahashi T, Ueno NT, Hung MC, and Esteva FJ (2004) P27(kip1) down-regulation is associated with trastuzumab resistance in breast cancer cells. Cancer Res 64:3981-3986.

Pandolfi PP (2004) Breast cancer-loss of PTEN predicts resistance to treatment. N Engl J Med 351:2337-2338.

Tseng PH, Lin HP, Zhu J, Chen KF, Hade EM, Young DC, Byrd JC, Grever M, Johnson K, Druker BJ, et al. (2005) Synergistic interactions between imatinib mesylate and the novel phosphoinositide-dependent kinase-1 inhibitor OSU-03012 in overcoming imatinib mesylate resistance. Blood 105:4021-4027.

Yakes FM, Chinratanalab W, Ritter CA, King W, Seelig S, and Arteaga CL (2002) Herceptin-induced inhibition of phosphatidylinositol-3 kinase and Akt Is required for antibody-mediated effects on p27, cyclin D1, and antitumor action. Cancer Res 62:4132-4141.

Yarden Y (2001) Biology of HER2 and its importance in breast cancer. Oncology 61 (Suppl 2):1-13.

Zheng FF, Kuduk SD, Chiosis G, Munster PN, Sepp-Lorenzino L, Danishefsky SJ, and Rosen N (2000) Identification of a geldanamycin dimer that induces the selective degradation of HER-family tyrosine kinases. Cancer Res 60:2090-2094. Zhu J, Huang JW, Tseng PH, Yang YT, Fowble J, Shiau CW, Shaw YJ, Kulp SK, and Chen CS (2004) From the cyclooxygenase-2 inhibitor celecoxib to a novel class of 3-phosphoinositide-dependent protein kinase-1 inhibitors. Cancer Res 64:43094318 .

Address correspondence to: Dr. Ching-Shih Chen, at College of Pharmacy 336 Parks Hall, The Ohio State University, 500 West 12th Avenue, Columbus, OH 43210-1291. E-mail: chen.844@osu.edu 